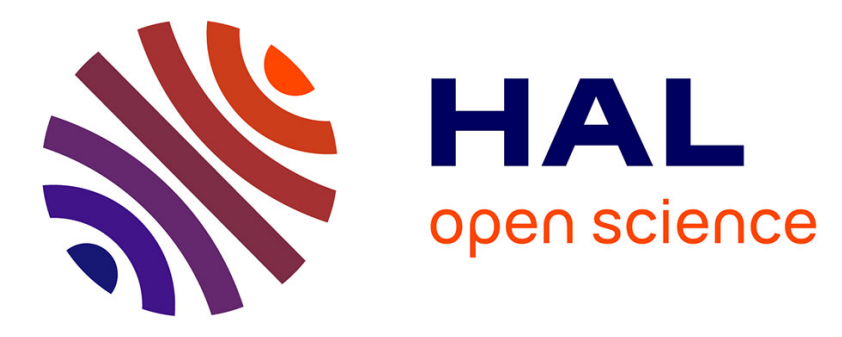

\title{
Model of Ni-63 battery with realistic PIN structure
}

Charles Munson, Muhammad Arif, Jeremy Streque, Belahsene Sofiane, Anthony Martinez, Abderrahim Ramdane, Youssef El Gmili, Jean-Paul Salvestrini, Paul L. Voss, Abdallah Ougazzaden

\section{To cite this version:}

Charles Munson, Muhammad Arif, Jeremy Streque, Belahsene Sofiane, Anthony Martinez, et al.. Model of Ni-63 battery with realistic PIN structure. Journal of Applied Physics, 2015, 118 (10), pp.105101. 10.1063/1.4930870 . hal-01203762

\section{HAL Id: hal-01203762 https://hal.science/hal-01203762}

Submitted on 1 Feb 2022

HAL is a multi-disciplinary open access archive for the deposit and dissemination of scientific research documents, whether they are published or not. The documents may come from teaching and research institutions in France or abroad, or from public or private research centers.
L'archive ouverte pluridisciplinaire HAL, est destinée au dépôt et à la diffusion de documents scientifiques de niveau recherche, publiés ou non, émanant des établissements d'enseignement et de recherche français ou étrangers, des laboratoires publics ou privés. 


\title{
Model of Ni-63 battery with realistic PIN structure
}

\author{
Charles E. Munson IV, ${ }^{1,2}$ Muhammad Arif, ${ }^{1,3}$ Jeremy Streque, ${ }^{1}$ Sofiane Belahsene, ${ }^{4}$ \\ Anthony Martinez, ${ }^{4}$ Abderrahim Ramdane, ${ }^{4}$ Youssef El Gmili, ${ }^{1}$ Jean-Paul Salvestrini, ${ }^{1,3}$ \\ Paul L. Voss, ${ }^{1,2}$ and Abdallah Ougazzaden ${ }^{1,2, a)}$ \\ ${ }^{1}$ Georgia Tech Lorraine, Georgia Tech-C.N.R.S., UMI2958, 2-3 rue Marconi, 57070 Metz, France \\ ${ }^{2}$ School of Electrical and Computer Engineering, Georgia Institute of Technology, 777 Atlantic Drive NW, \\ 30332-0250 Atlanta, USA \\ ${ }^{3}$ Université de Lorraine, CentraleSupélec, LMOPS, EA 4423, 2 rue E. Belin, 57070 Metz, France \\ ${ }^{4}$ Laboratory for Photonics and Nanostructures, CNRS, Route de Nozay, 91460 Marcoussis, France
}

(Received 30 June 2015; accepted 31 August 2015; published online 11 September 2015)

\begin{abstract}
$\mathrm{GaN}$, with its wide bandgap of $3.4 \mathrm{eV}$, has emerged as an efficient material for designing highefficiency betavoltaic batteries. An important part of designing efficient betavoltaic batteries involves a good understanding of the full process, from the behavior of the nuclear material and the creation of electron-hole pairs all the way through the collection of photo-generated carriers. This paper presents a detailed model based on Monte Carlo and Silvaco for a GaN-based betavoltaic battery device, modeled after Ni-63 as an energy source. The accuracy of the model is verified by comparing it with experimental values obtained for a GaN-based $\mathrm{p}-\mathrm{i}-\mathrm{n}$ structure under scanning electron microscope illumination. (C) 2015 AIP Publishing LLC.
\end{abstract}

[http://dx.doi.org/10.1063/1.4930870]

\section{INTRODUCTION}

While electronic devices and battery size requirements have been continually shrinking over the last several decades, energy density requirements have steadily been increasing at the same time. Classic, chemical-based batteries unfortunately do not scale well with size, and thus do not perform well when shrunk down to power micro-sized devices. Additionally, these batteries have comparably short lifetimes, or must be constantly recharged.

Nuclear batteries can provide a high energy density with a long half-life (100 years for Ni-63 (Ref. 1)), and thus do not need to be refueled or recharged as often. Ni-63 beta particles are stopped within several micrometers of travel through solids and, thus, batteries designed with this fuel source can be easily and safely packaged with minimal danger.

Theoretical research into using $\mathrm{GaN}$ as a betavoltaic battery has been undertaken over the past few decades. Polikarpov and Yakimov $^{2}$ published simulation results for Ni-63 like particles with a Scanning Electron Microscope (SEM) at various angles into the material and found the energy loss at various depths within $\mathrm{GaN}$ and Si. Simulations done by Zuo et al. ${ }^{3}$ were done with Ni-63 and Pm-147 on GaN and Si materials, showing expected voltages and currents. Additionally, San et al. ${ }^{4}$ published simulation results on a Ni-63 Schottky GaN device, estimating it could achieve a Power Conversion Efficiency (PCE) of 2.25\%. In addition to GaN-based betavoltaic batteries using Ni-63, theoretical research has also been done using various other radioactive materials ${ }^{5,6}$ and semiconductor materials, ${ }^{7-9}$ with expected performances ranging from poor (high electronhole pair recombination rates) to nearly $5 \%$ efficiency.

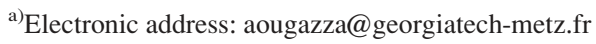

Similarly, experimental research has also been done in this field. A group comprised of Lu et al. ${ }^{10}$ created a GaNbased PIN device that boasted a PCE of 1.6\%. Devices using iron-doping of the intrinsic region of a GaN-based PIN similarly yielded results ranging from $0.98 \%$ to $2.7 \% .^{11-13}$ Likewise, Ni-63 experimental research using non GaN-based materials have been performed, ${ }^{1,14,15}$ yielding potentially promising results after changes could be possibly made to the designs in the future.

An important part of designing devices is having a deep understanding of how the processes involved in the device will work. In order to effectively design a betavoltaic battery, the processes involved beginning from the behavior of the nuclear material all the way through the current extraction from the device itself must be well understood. Currently, there are no comprehensive publications that detail this full process, while additionally verifying the mathematical models via experimentation to ensure accuracy. In this paper, we will show the process of representing all of the involved phenomena relating to betavoltaic batteries, including emission from the radioactive source, losses through contact metals, absorption into the semiconductor materials, and collection of betacurrent through contacting pads while taking parasitic resistances into account. This design procedure will allow us to take any given PIN device and predict the real-world performance of the device under beta particle bombardment (Ni-63), given only the I-V characteristics and physical properties of the device (such as the $\mathrm{p} / \mathrm{i} / \mathrm{n}$ region widths and doping levels).

In the following sections, we will detail the process. Section II will discuss the model, including specifications of the physical device that we sought to mimic, a SEM model detailing the behavior of injected electrons, and carrying this SEM model into a device model to predict the real-world electrical behavior and performance assuming the device 
was perfect (no defects or parasitic resistances). Section III will then describe the modeling of device defects as an ideal diode and series/shunt resistances and will discuss how this final model matches up with the experimental data performed with a real device under SEM current injection in order to verify the accuracy of the model. Finally, in Section IV, we will describe how to use the model in order to help design a betavoltaic device and estimate the real-world performance of such a device.

\section{MODEL}

\section{A. Device structure}

In order to create an accurate model, a real-life device had to be emulated. In Figure 1, we can see the $4 \mathrm{~mm} \times 4 \mathrm{~mm}$ area PIN device used for experimental analysis. It includes a $40 \mathrm{~nm}$ current spreading layer on top of the device $(20 \mathrm{~nm}$ of gold and $20 \mathrm{~nm}$ of palladium). This is followed by a $150 \mathrm{~nm}$ $\mathrm{p}-\mathrm{GaN}$ region (carrier concentration of $5 \times 10^{17} / \mathrm{cm}^{-3}$ ) and a $600 \mathrm{~nm}$ i-GaN region (estimated to have an unintentionally doped carrier concentration of around $3 \times 10^{16} / \mathrm{cm}^{-3}$ ). The rest of the device is n-type $\mathrm{GaN}$ with a carrier concentration of around $3 \times 10^{18} / \mathrm{cm}^{-3}$.

The inset shows a SEM image of the actual device from the top view along with contacting probes touching the ncontacts and p-contacts. The p-GaN mesa is also highlighted and can clearly be seen in this image.

Figure 2 shows the experimental device under an optical microscope. Clearly visible are the $\mathrm{n}-\mathrm{GaN}$ contacts and large $\mathrm{p}-\mathrm{GaN}$ contact. The dark gray pad for the $\mathrm{p}-\mathrm{GaN}$ contact is also visible around the $\mathrm{p}-\mathrm{GaN}$ contact, while the $\mathrm{p}-\mathrm{GaN}$ mesa shows the separation between the p-GaN and n-GaN regions.

\section{B. Ni-63-like beta particle emission}

The first part of the model deals with mimicking Ni-63 emissions with an e-beam current. Since the e-beam can only emit at a single electron energy, we had to choose which energy would most accurately represent the energy of beta particles coming from Ni-63. It is well known that the most

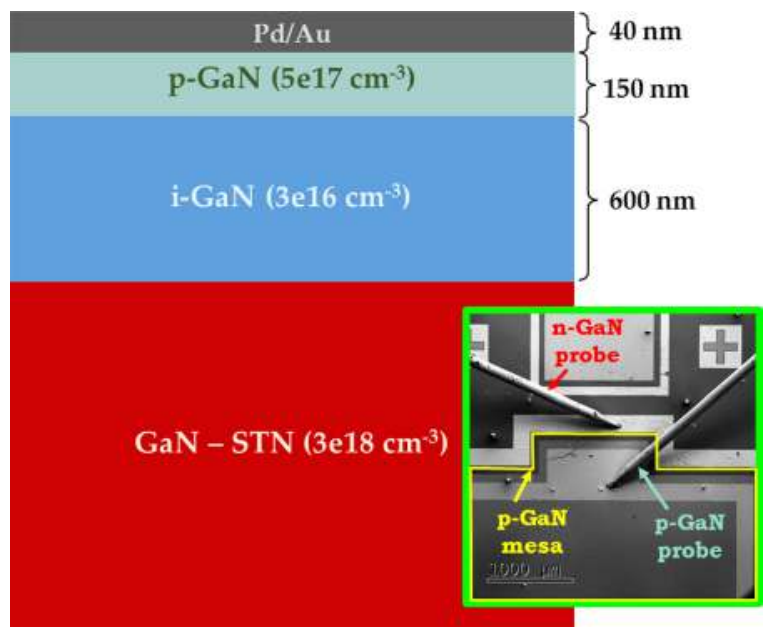

FIG. 1. Experimental device structure. Inset: SEM image of device with n$\mathrm{GaN}$ and $\mathrm{p}-\mathrm{GaN}$ probes, as well as p-GaN mesa.

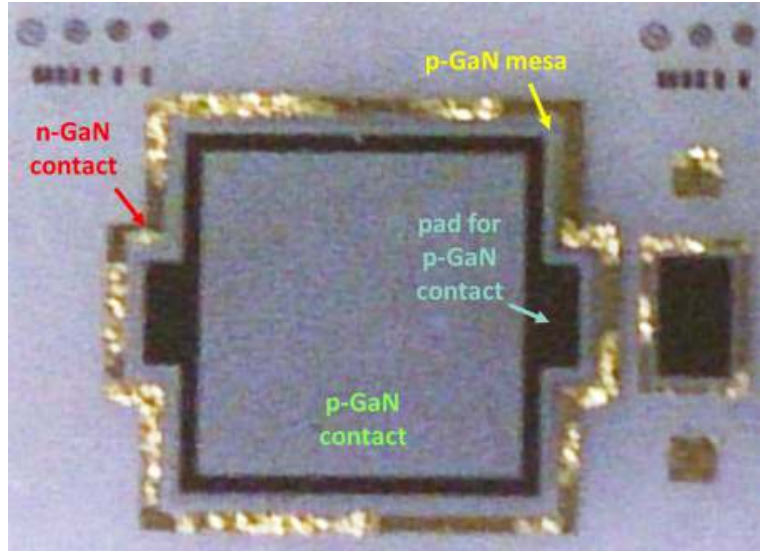

FIG. 2. Photograph of experimental device.

probable energy released from Ni-63 is around $17 \mathrm{keV}$ and quickly declines in probability when the energies fall outside the range of $5 \mathrm{keV}$ and $50 \mathrm{keV}$. $^{9}$

As can be seen in Figure 3, a Probability Density Function (PDF) of the electron energy absorption profile was calculated based on the CASINO 2 Monte Carlo simulations for $17 \mathrm{keV} .{ }^{16}$ The integrated area under the curve for a given depth range provides the percentage of electron energy that is absorbed in that depth range, and the area under the entire curve (including the $40 \mathrm{~nm}$ metal spreading layer, not shown) will add up to $100 \%$. Note that this does not take into account backscattering of electrons off of the sample surface; the e-beam source currents used in this paper are the specimen currents, which ignores the effects of backscattered, secondary, and transmitted electrons.

Electron energies absorbed within the $40 \mathrm{~nm}$ of metal spreading layer are assumed to be lost, and so are not included in the absorption model (though they account for $8.6 \%$ of electron energy absorption). This absorption model was then later used in the device simulations to determine the electron-hole pairs that would be generated from such a profile (and consequently the current that would be generated). We can see here that around $22 \%$ of the energy is lost from penetration through the metal spreading layer (not shown) and $\mathrm{p}-\mathrm{GaN}$ regions (8.6\% and $13.7 \%$, respectively) of the device, while $51.4 \%$ of the total energy will fall within the intrinsic GaN region. The remaining $26.3 \%$ of the energy is lost in the $\mathrm{n}-\mathrm{GaN}$ region and substrate.

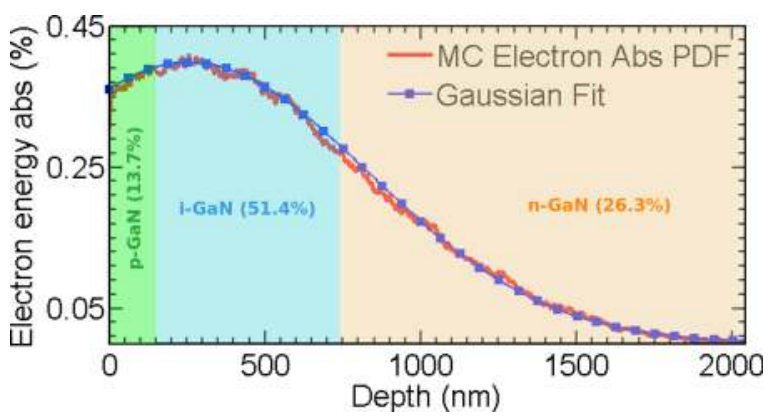

FIG. 3. Electron energy absorption PDF at $17 \mathrm{keV}$ SEM, first $40 \mathrm{~nm}$ of spreading layer not included. 
Figure 4 shows the distribution of beta particle energy absorption in certain regions of the device versus electron energy. As expected, lower electron energies are almost completely absorbed by the current spreading layer, while electron energies from around $10 \mathrm{keV}$ to $22 \mathrm{keV}$ are predominantly absorbed within the i-GaN region of the device. Higher electron energies, above $22 \mathrm{keV}$, are lost to absorption mostly within the $\mathrm{n}-\mathrm{GaN}$ region of the device. Note again that this figure does not show losses due to backscattering from the device surface but is intended to show which regions of the device are absorbing the most amounts of energy at different electron energies.

\section{Mapping beta particles to photons}

Now that a model of the behavior of beta particle-like absorption into the GaN PIN had been created using Monte Carlo simulations, detailed device model simulations had to be done.

For the device simulations, we used Silvaco TCAD software. Parameters to use in the simulation for the GaN materials involved were taken from Mnatsakanov et al. ${ }^{17}$ and Vurgaftman and Meyer. ${ }^{18}$ All other parameters for the simulated device were taken from measurements made directly from the experimental device (minority carrier concentrations, region thicknesses, etc.).

Based on literature, ${ }^{19}$ it has been shown that for GaNbased materials, the maximum betavoltaic current efficiency is approximately $27 \%$. Because the device simulations do not take this into account (it assumes the particles are photons, whose frequency we have chosen to be optimal for absorption in $\mathrm{GaN}$ ), we multiply the efficiency of the device by 0.27 .

Finally, an absorption profile for the SEM electrons needed to be imported into Silvaco software for device simulation. Because this software does not support beta particles but only photons, the absorption model of the electrons had to be accurately mapped to the photon model in Atlas. In order to achieve this, a PDF was fitted to the Monte Carlo

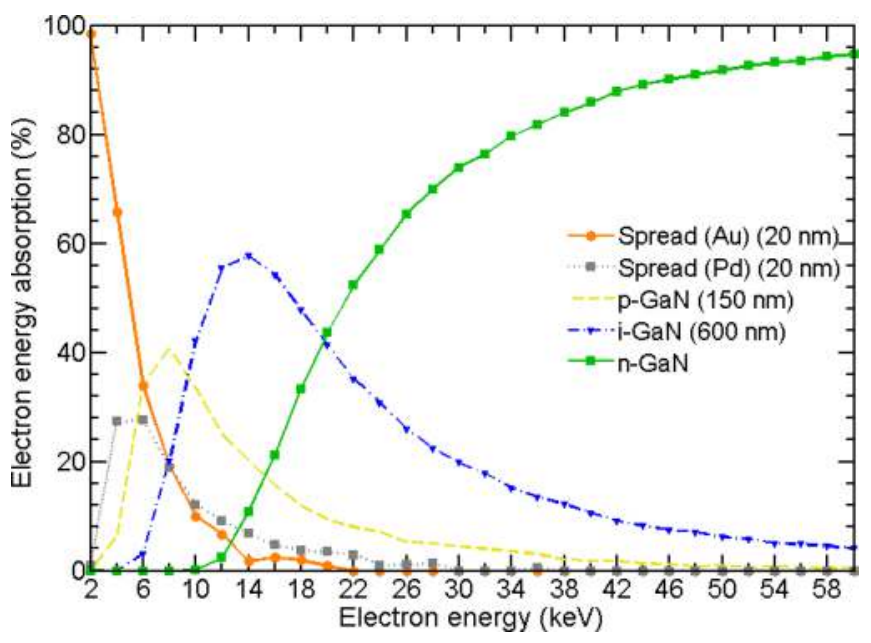

FIG. 4. Distribution of electron energy absorption versus electron energy across various device regions. simulation data from Figure 3, represented mathematically in the following equation:

$$
\text { Absorption rate } \sim \frac{1}{\sigma \times \sqrt{2 \pi}} \times \exp \left(-\frac{(y-\mu)^{2}}{2 \times \sigma^{2}}\right),
$$

where $\mathrm{y}$ is the vertical depth down into the GaN material in nanometers, $\mu$ is the mean value for our PDF $(256.53 \mathrm{~nm})$, and $\sigma$ is the standard deviation $(576 \mathrm{~nm})$. Note that this equation gives us the shape of absorption and an arbitrary magnitude. The magnitude is determined based on the expected device efficiency (for a perfect device) with losses due to the current spreading layer (already taken into account previously, $\sim 8 \%$ loss), beta particle efficiency, and losses in the $\mathrm{p}-\mathrm{GaN}$ and $\mathrm{n}-\mathrm{GaN}$ regions (whose electron-hole pairs will predominantly not be successfully collected).

\section{MATCHING MODEL TO EXPERIMENT}

In order to calibrate the simulator, the simulations need to be matched against real-world experimental results. We can estimate the power efficiency of a perfect PIN device with e-beam penetration at $17 \mathrm{keV}$ at this point

$$
\text { Max. power efficiency }=0.514 \times 0.27 \rightarrow 0.1388,
$$

where $51.4 \%$ is the amount of electron energy falling within the i-GaN region (which has the potential to be collected, as described in Section II B) and 27\% is the maximum betavoltaic current efficiency for GaN (as described in Section II C). This leads to a highest possible PCE of around 14\%, not taking into account device defects/traps and contacting deficiencies (which will be taken into account later).

The device simulations are run with perfect, defect-less device materials, while scaling the magnitude of the absorption rate given in Eq. (1), until the simulation results give the device efficiency predicted in Eq. (2). At this point, defects and traps can then be added to the model (if they are known) in order to better calibrate the expected device performance. The corresponding dark and illuminated I-V curves from the simulated device will next be used for our series and shunt resistance model, allowing us to model the effects related to contact inefficiencies.

\section{A. Series and shunt resistance model}

In order to better take into account defects introduced into the device by recombination and issues introduced by the contacts, the ideality of the real-world version needs to be matched to the simulated device. We used this information to scale the simulated dark I-V curve to more accurately mimic the experimental device using the following ideal diode equation:

$$
I d=I s \times\left\{\exp \left(\frac{q \times V d}{n \times k \times T}\right)-1\right\},
$$

where Is is the saturation current, $\mathrm{q}$ is the electron charge, $\mathrm{n}$ the ideality factor, $\mathrm{k}$ is Boltzmann's constant, and $\mathrm{T}$ is the temperature $(300 \mathrm{~K})$. 


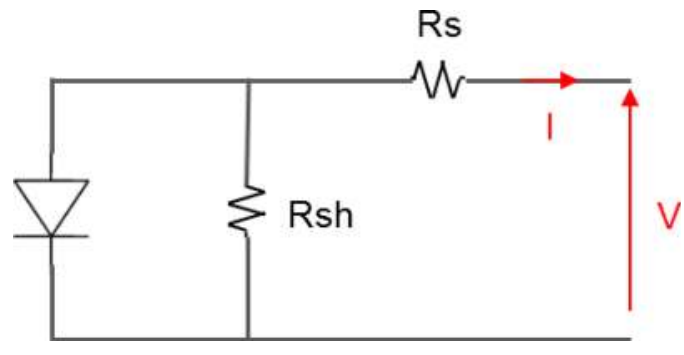

FIG. 5. Single diode series and shunt resistance model.

Additional defects are introduced by the contacts that can be modeled as shunt and series resistances that were not already taken into account with the ideality factor (n), as can be seen in Eqs. (4) and (5) and Figure 5

$$
\begin{gathered}
I=I d+\frac{V d}{R s h}, \\
V=\left(1+\frac{R s}{R s h}\right) \times V d+I d \times R s .
\end{gathered}
$$

Here, Rs represents the total series resistance of the device, Rsh is the total shunt resistance, and Id and Vd are the ideal IV-curve current and voltage, respectively.

The results of this model match up well with effects previously described in literature, ${ }^{20}$ where the shunt resistance has a large impact in the lower voltage range, while effects due to series resistance dominate in the higher voltages (although we obtained the model by first modeling the device as an ideal diode with measured ideality and then applied the series and shunt resistance models). The threshold voltage drop with increasing series resistance also matches up well with literature studying the effects of these resistances. $^{21}$

Note that this model can be further refined by using a two- or three-diode version instead of only one diode (as in Figure 5), depending on the level of accuracy needed for the IV-curves. Additionally, an illuminated model can be made even more accurate by matching it to experimental IVcurves under similar-power illumination (such as SEM illumination to improve the quality of a Ni-63 model).

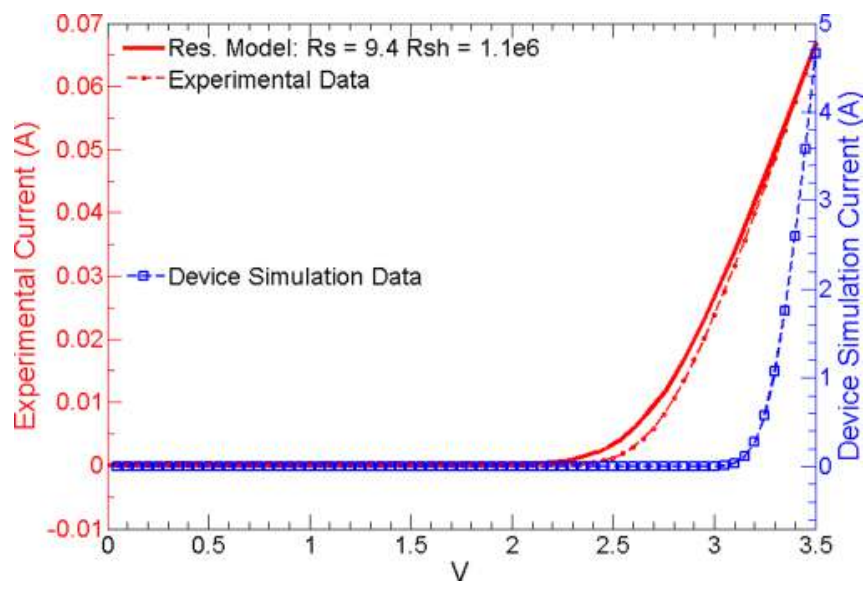

FIG. 6. Dark I-V fitting curves—simulated vs experimental data.

\section{B. Experimental data and model comparison}

In Figure 6, we see the original, device simulation IVcurve as the dashed blue line. The red, dashed line is the experimental dark IV-curve with the device undergoing no beta particle bombardment, while the solid red line is the ideal diode equation scaled to take series and shunt resistances (of $9.4 \Omega$ and $1.1 \mathrm{M} \Omega$, respectively) into account. These values were specifically chosen to best fit the experimental results.

Now that we have created a model that also reflects the inefficiencies introduced by the contacts, we can model the beta-illuminated conditions of the device. The simplest way to do this is to take the predicted Isc by the device simulations for a given input power and combine it with the series and shunt resistance model's dark I-V curve, as described previously.

These simulation results can then be compared to results obtained experimentally. For the following experiments, the sample was mounted onto a commercial holder with two probes for the e-beam bombardment measurements. The probes on the sample were connected to an output circuit with a Keithley 4200 for measuring the I-V behavior of the device during bombardment. Several beam energies were tested and the ones with values close to Ni-63 were then selected, as presented in Figure 7.

Here, we can see the green dashed line is the experimental IV-curve with $7.3 \mu \mathrm{W}$ of energy introduced via e-beam (at $17 \mathrm{keV}$ ), the blue dashed line is experimental with 29 $\mu \mathrm{W}$, and the purple dashed line is experimental with $80 \mu \mathrm{W}$. The solid, colored lines are the final, simulated approximations of the device for each respective input energy. The dashed gray line shows the raw device simulation data for 80 $\mu \mathrm{W}$ of power introduced, which assumes a near-perfect device, before the resistance model is added-compare this with the solid, purple line, which additionally adds the series and shunt resistance model.

We can also see this expressed numerically in Table I. In the ideal simulated diode (directly from the device simulations), we have a high open-circuit voltage mainly because it has no defects at all, not even from parasitic resistances in

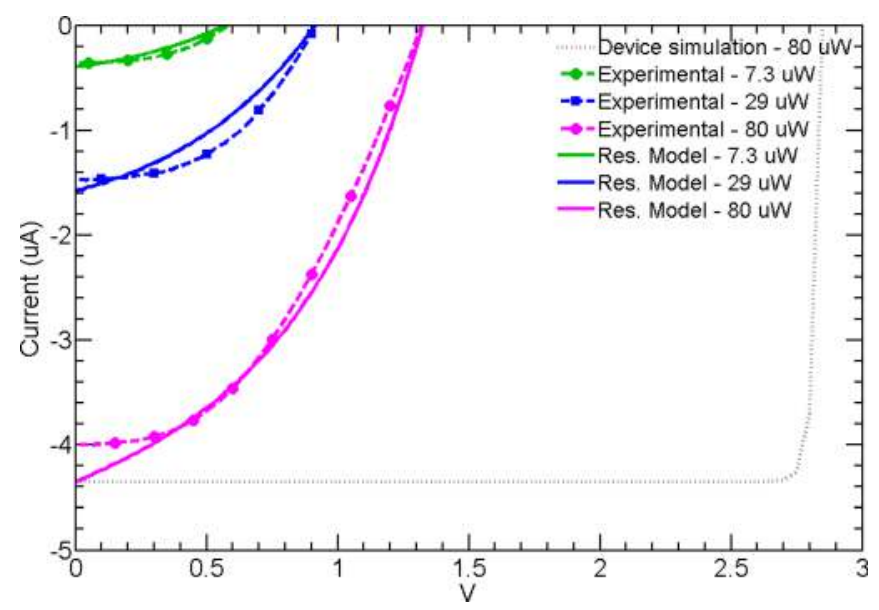

FIG. 7. Comparison of modeled illuminated curves and experimental illuminated curves. Device simulation illuminated curve at $80 \mu \mathrm{W}$ provided for comparison to resistance model. 
the contacts. We can see the difference after adding the ideality and resistance models to the ideal simulated diode, where the open-circuit voltage drops from $3 \mathrm{~V}$ to $1.3 \mathrm{~V}$. Both the experimental and simulated resistance models expect around $4 \mu \mathrm{A}$ at $0 \mathrm{~V}$ applied to the device and $80 \mu \mathrm{W}$ of power from the e-beam. With an open-circuit voltage of around $1.3 \mathrm{~V}$, the model and experiments show a device power expected to be over $2 \mu \mathrm{W}$ (and thus an efficiency of around $3 \%$ ).

\section{USAGE OF MODEL IN PRACTICE}

Now that we have shown all of the simulated pieces from electron emission to betacurrent collection from the device, we will now describe the process of using this full model to aid in the designing of a betavoltaic device.

\section{A. Choose illumination/absorption model}

Using Monte Carlo simulations, a good estimate of the electron absorption behavior can be determined for a given material. In this paper, we discussed SEM injection of 17 $\mathrm{keV}$ electrons straight down into GaN material and provided an absorption profile from these simulations (see Eq. (1)). This process can be repeated for other materials (e.g., silicon or $\mathrm{SiC}$ ) to obtain their respective profiles, depending on which materials are to be used for the desired device. In this paper, we also mention usage of a Ni-63 model, which will be discussed in detail in a future paper. Using this Ni-63 model, for instance, a good estimate for Ni-63 absorption into a device can be determined without needing to perform expensive and burdensome Ni-63 tests experimentally.

\section{B. Build device model}

Now that an absorption model has been chosen, this model can be put into device simulation software. A proposed device can be constructed in Silvaco with a desired material and dimensions and, when coupled with this beta absorption profile and material efficiency (as discussed in Eq. (2)), an accurate Isc value can be obtained. Note that this will not give an accurate device Voc (and thus the I-V curve will also not be accurate) because this model assumes a nearperfect device with no major defects or parasitic resistances (though they can be included if an even more accurate Isc value is desired). If a full I-V curve and Voc are required, we must continue to the next step.

TABLE I. Illuminated electrical characteristics at $80 \mu \mathrm{W}$ power deposited in the sample. "Sim-Ideal" shows the expected performance based on device simulations, "Sim-Res" is after the resistance model has been applied to the device simulation model, and "Exp" is the experimental results.

\begin{tabular}{lccc}
\hline \hline & Sim-Ideal & Sim-Res & Exp \\
\hline Isc $(\mu \mathrm{A})$ & 4.34 & 4.34 & 4.03 \\
Voc $(\mathrm{V})$ & 3.03 & 1.3 & 1.3 \\
Device power $(\mu \mathrm{W})$ & 12.43 & 2.34 & 2.33 \\
Fill factor $(\%)$ & 94.5 & 41.5 & 44.4 \\
PCE $(\%)$ & 15.54 & 2.93 & 2.91 \\
\hline \hline
\end{tabular}

\section{Get I-V curve experimentally from similar device}

To get accurate Voc and I-V curve shape, we first need an experimental dark I-V curve from a similar device to what was obtained in the device simulation design. This can be done by custom-growth of the simulated device or by purchasing an already-prepared device commercially. The important factor is that the device will have the same material and contact properties as the final betavoltaic device. The model can be even further refined if light or SEM bombardment is done on this device and experimental I-V curves are taken for various power levels, allowing various types of absorption models to match even more closely for the model.

\section{Use resistance transformation}

In order to aid in better fitting the experimental and simulated curves to each other, the I-V curve data can be modeled using the ideal diode model described in Eq. (3)) via a best-fit method. This step will allow us to take into account device inefficiencies via the ideality parameter. Finally, using the resistance transformation provided in Eq. (4), convert the dark Silvaco-based I-V curve diode model into the dark experimental I-V curve diode model, resulting in Rs/Rsh value estimates and a good estimation of the I-V curve for the device under the chosen illumination/absorption model. The end result here is that we now have an I-V curve that matches a particular device and can also accurately predict the Voc and Isc values for the chosen illumination/absorption.

\section{E. Ni-63 absorption model example}

Using the Ni-63 absorption profile-to be released in more detail in a future publication-under $13.6 \mu \mathrm{W}$ of power (5 GBq of activity) instead of the SEM profile, we get the results seen in Table II. This model assumes a stacked PIN design with energy collected in both a top and bottom PIN device; it was created by compiling various Monte Carlo simulations of differing electron emission angles and energies in order to best match the emission profile and behavior of Ni-63 as possible. This model takes into account backscatter off of the sample surface, as well as the self-absorption of electrons within the Ni-63 source. With an ideal diode (no defects or parasitic resistances), we expect a high opencircuit voltage of $2.95 \mathrm{~V}$, while this drops to only $0.44 \mathrm{~V}$ when we add in defects due to ideality and parasitic resistances. This is responsible for dropping the efficiency from $5.7 \%$ to only $0.5 \%$, resulting in an output power of around $68 \mathrm{nW}$.

TABLE II. Illuminated electrical characteristics with simulated Ni-63 source at $13.6 \mu \mathrm{W}$ power.

\begin{tabular}{lcc}
\hline \hline & Sim-Ideal & Sim-Res \\
\hline Isc $(\mu \mathrm{A})$ & 0.28 & 0.28 \\
Voc $(\mathrm{V})$ & 2.95 & 0.44 \\
Device power $(\mu \mathrm{W})$ & 0.78 & 0.068 \\
Fill factor $(\%)$ & 94.3 & 54.7 \\
PCE $(\%)$ & 5.7 & 0.5 \\
\hline \hline
\end{tabular}


TABLE III. Illuminated electrical characteristics with Ni-63 source at 50 $\mathrm{nW}$ power.

\begin{tabular}{lccc}
\hline \hline & Sim-Ideal & Sim-Res & Exp $^{13}$ \\
\hline Isc (pA) & 617.6 & 617.6 & 568 \\
Voc (V) & 2.58 & 1.7 & 1.64 \\
Device power (nW) & 1.59 & 1.05 & 0.93 \\
Fill factor (\%) & 54.8 & 54.3 & 53 \\
PCE (\%) & 1.74 & 1.14 & 0.98 \\
\hline \hline
\end{tabular}

Next, the model was matched to an experiment done by Cheng et al. ${ }^{13}$ in order to test its accuracy with a device that experimentally underwent Ni-63 bombardment. This experiment was chosen in particular, because it matches closely to the specifications of the project that we are working on and developing a device for and is taken as state-of-the-art for GaN-based, Ni-63-sourced betavoltaic batteries.

In Table III, we see that, after our resistance model is applied to the device simulations of their device, the model's expected efficiency is $1.14 \%$ - only $0.16 \%$ higher than the experimental efficiency of $0.98 \%$. Note that, since the material properties related to the iron doping of their intrinsic region were not fully detailed, values from literature ${ }^{22,23}$ were used instead. The electron mobility of their iron-doped material was also assumed to be state-of-the-art quality, which explains why the model overestimated the expected short-circuit current and thus device power.

\section{CONCLUSION}

In conclusion, we have demonstrated a betavoltaic model that involves emission of the most probable beta particle energy from Ni-63 (17 keV) directly into a GaN-based PIN device. The model takes into account losses through contacting metals, losses by energy absorption efficiency of electrons into GaN-like materials, losses due to electron-hole pairs that are created outside of the intrinsic region of the device, and defects due to recombination and parasitic resistances (ideality factor and series-shunt resistances).

We have demonstrated that the model matches well with experimental results of a fabricated device in dark conditions and under SEM-illumination. We have also demonstrated that the model matches well with Ni-63 bombardment results from literature. In the end, we expect that the device could operate with an efficiency and short-circuit current that are within a $10 \%$ margin of error.

\section{ACKNOWLEDGMENTS}

This work was supported by the Agence Nationale de la Recherche of France as part of the BATGAN project.

${ }^{1}$ B. Ulmen, P. D. Desai, S. Moghaddam, G. H. Miley, and R. I. Masel, "Development of diode junction nuclear battery using Ni-63," J. Radioanal. Nucl. Chem. 282(2), 601-604 (2009).

${ }^{2}$ M. A. Polikarpov and E. B. Yakimov, "Application of a scanning electron microscope in simulating a beta-emission-induced current," J. Surf. Invest.: X-Ray, Synchrotron Neutron Tech. 7(1), 81-84 (2013).
${ }^{3}$ G. P. Zuo, J. L. Zhou, and G. T. Ke, "A simple theoretical model for 63 ni betavoltaic battery," Appl. Radiat. Isot. 82(0), 119-125 (2013).

${ }^{4}$ H. San, S. Yao, X. Wang, Z. Cheng, and X. Chen, "Design and simulation of GaN based Schottky betavoltaic nuclear micro-battery," Appl. Radiat. Isot. 80, 17-22 (2013).

${ }^{5}$ F. K. Manasse, J. J. Pinajian, and A. N. Tse, "Schottky-barrier betavoltaic battery," IEEE Trans. Nucl. Sci. 23(1), 860-870 (1976).

${ }^{6}$ T. Wacharasindhu, J. W. Kwon, D. E. Meier, and J. D. Robertson, "Radioisotope microbattery based on liquid semiconductor," Appl. Phys. Lett. 95(1), 014103 (2009).

${ }^{7}$ C. Hai-Yang, J. Lan, and L. Da-Rang, "Measurement of beta particles induced electron-hole pairs recombination in depletion region of GaAs PN junction," Chin. Phys. Lett. 28(5), 058101 (2011).

${ }^{8}$ T. XiaoBin, D. Ding, L. YunPeng, and C. Da, "Optimization design and analysis of Si-Ni-63 betavoltaic battery," Sci. China: Technol. Sci. 55(4), 990-996 (2012).

${ }^{9}$ S. Yao, Z. Song, X. Wang, H. San, and Y. Yu, "Design and simulation of betavoltaic battery using large-grain polysilicon," Appl. Radiat. Isot. 70(10), 2388-2394 (2012).

${ }^{10} \mathrm{M}$. Lu, G. Wang, and C. Yao, "Gallium nitride for nuclear batteries," in Materials for Environmental Protection and Energy Application, Advanced Materials Research Vol. 343-344, edited by D. Wang (Trans Tech Publications Ltd, Laublsrutistr 24, CH-8717 Stafa-Zurich, Switzerland, 2012), Pts. 1 and 2, pp. 56-61; in International Conference on Materials for environmental Protection and Energy Application (MEPEA 2011), Kuala Lumpur, Malaysia, 27-28 September 2011.

${ }^{11}$ Z. J. Cheng, H. S. San, Z. H. Feng, B. Liu, and X. Y. Chen, "High opencircuit voltage betavoltaic cell based on GaN pin homojunction," Electron. Lett. 47(12), 720-721 (2011).

${ }^{12}$ C. Zai-Jun, S. Hai-Sheng, C. Xu-Yuan, L. Bo, and F. Zhi-Hong, "Demonstration of a high open-circuit voltage GaN betavoltaic microbattery," Chin. Phys. Lett. 28(7), 078401 (2011).

${ }^{13}$ Z. Cheng, X. Chen, H. San, Z. Feng, and B. Liu, "A high open-circuit voltage gallium nitride betavoltaic microbattery," J. Micromech. Microeng. 22(7), 074011 (2012); in 22nd Micromechanics and Microsystems Europe Workshop (MME), Toensberg, Norway, 19-22 June 2011.

${ }^{14}$ Y. Liu, R. Hu, Y. Yang, G. Wang, S. Luo, and N. Liu, "Investigation on a radiation tolerant betavoltaic battery based on schottky barrier diode," Appl. Radiat. Isot. 70(3), 438-441 (2012).

${ }^{15}$ L. YunPeng, T. XiaoBin, X. ZhiHeng, H. Liang, W. Peng, and C. Da, "Optimization and temperature effects on sandwich betavoltaic microbattery," Sci. China: Technol. Sci. 57(1), 14-18 (2014).

${ }^{16}$ D. Drouin, A. R. Couture, D. Joly, X. Tastet, V. Aimez, and R. Gauvin, "Casino v2. 42-a fast and easy-to-use modeling tool for scanning electron microscopy and microanalysis users," Scanning 29(3), 92-101 (2007).

${ }^{17}$ T. T. Mnatsakanov, M. E. Levinshtein, L. I. Pomortseva, S. N. Yurkov, G. S. Simin, and M. A. Khan, "Carrier mobility model for GaN," Solid-State Electron. 47(1), 111-115 (2003).

${ }^{18}$ I. Vurgaftman and J. R. Meyer, "Band parameters for nitrogen-containing semiconductors," J. Appl. Phys. 94(6), 3675-3696 (2003).

${ }^{19}$ L. C. Olsen, Review of Betavoltaic Energy Conversion in NASA Conference Publication (NASA, 1993), pp. 256-256.

${ }^{20}$ M. Barbato, M. Meneghini, A. Cester, G. Mura, E. Zanoni, and G. Meneghesso, "Influence of shunt resistance on the performance of an illuminated string of solar cells: Theory, simulation, and experimental analysis," IEEE Trans. Device Mater. Reliab. 14(4), 942-950 (2014).

${ }^{21}$ J. Zhang, S.-T. Lee, and B. Sun, "Effect of series and shunt resistance on organic-inorganic hybrid solar cells performance," Electrochim. Acta 146, 845-849 (2014)

${ }^{22}$ H. Witte, K. Fluegge, A. Dadgar, A. Krtschil, A. Krost, and J. Christen, "Deep defects in Fe-doped GaN layers analysed by electrical and photoelectrical spectroscopic methods," in GaN and Related Alloys, Materials Research Society Symposium Proceedings, edited by H. M. Ng, M. Wraback, K. Hiramatsu, and N. Grandjean (Materials Research Society, 506 Keystone Drive, Warrendale, PA 15088-7563, USA, 2003), Vol. 798, pp. 575-580; in Symposium on GaN and Related Alloys held at the MRS Fall Meeting, Boston, MA, 01-05 December 2003.

${ }^{23}$ A. Y. Polyakov, N. B. Smirnov, A. V. Govorkov, V. I. Vdovin, A. V. Markov, A. A. Shlensky, E. Prebble, D. Hanser, J. M. Zavada, and S. J. Pearton, "Properties of Fe-doped, thick, freestanding GaN crystals grown by hydride vapor phase epitaxy," J. Vac. Sci. Technol. B 25(3), 686-690 (2007). 